\title{
Our Study of Comparison of Oticon Digisonic and Cochlear Limited Implants in North India
}

\author{
Rohit Mehrotra ${ }^{1 *}$, Anubhaw ${ }^{2}$, Pankaj Srivastav ${ }^{3}$ and Rudresh Sharma ${ }^{4}$ \\ ${ }^{1}$ Professor of ENT, Mehrotra ENT Hospital, UP, India \\ ${ }^{2,3}$ Consultant, Mehrotra ENT Hospital, UP, India \\ ${ }^{4}$ Audiologist, Mehrotra ENT Hospital, UP, India
}

*Corresponding author: Rohit Mehrotra, Mehrotra ENT Hospital, Ashok Nagar, Kanpur, UP, India.

Received Date: June 06, 2020

Published Date: July 06, 2020

\begin{abstract}
Introduction: This study is a comparitive study between oticon Digisonic and Cochlear limited implants outcomes in 200 implant surgeries at late Dr S N Mehrotra charitable ENT foundation between 2016 to 2019.

Material and methods: 100 children of Digisonic and 100 of Cochlear Ltd who were implanted between 2016-2019 in Mehrotra ENT hospital, were included in the study. Outcomes of all the implanted children in both groups were analyzed. Scoring system for auditory performance, speech rehabilitation and quality of life were also taken into account for every child implanted. Results of both groups were compared.

Results: Highest CAP level attained in cochlear group was 10 whereas in Digisonic group 5. 23\% in Cochlear group had CAP level of 5 and above compared to only $1 \%$ in Digisonic group. 88\% of children in Cochlear group had highest SIR of 5 and 6 compared to $75 \%$ in Digisonic group. $87 \%$ of cochlear group had GCBI index greater than 80 (maximum benefit) compared to $41 \%$ of Digisonic group. Only $1 \%$ belongs to mild benefit in Cochlear group whereas in Digisonic 29\% in minimal benefit group.
\end{abstract}

Conclusion: Cochlear LTD group results were better when compared to Digisonic group in terms of hearing, speech and quality of life index. Digisonic implants had easier insertion. Cochlear proved to be better than Digisonic owing to superior hearing, speech and quality of life outcomes.

Keywords: Oticon digisonic; Cochlear limited; Electrode insertion; Outcomes

\section{Introduction}

Oticon is a hearing aid manufacturer based in Copenhagen, Denmark. It was founded in 1904 by Hans Demant, whose wife was hearing impaired. The company uses a management style known as "Spaghetti Organization" [1,2]. introduced by Lars Kolind [3-5]. The highly reliable cochlear implants in the Digisonic $® S P$ range enable surgeons to select the most appropriate solution for each patient, taking into account their cochlear anatomy, auditory nerve and any residual hearing.

The Digisonic $₫ S P$ implant houses a magnet and receiver within a single unit made of ceramic and titanium. This monobloc structure makes surgery easier because the implant can be simply slid under the skin and fixed into place using two titanium screws [6]. Furthermore, as the procedure is minimally invasive, patients can benefit from faster healing times and greater implant stability. Once in place, the casing's convex shape is designed to absorb any shock waves for greater impact resistance. The Digisonic $₫ S P$ cochlear implant range can be used with two different proven electrode arrays to suit the patient's anatomy.

The 20 stimulating electrodes of the Digisonic $® S P$ implant allow a very precise stimulation of the auditive nerve at an optimal speed 
of a maximum 24,000 pulses per second. This stimulation speed is intended to transmit essential information to you while reducing the energy consumption of the implant. As a result, the behind the ear that is paired with the Digisonic $囚 S P$ implant consumes only two Zinc-Air batteries for a battery life of 3 to 4 days (it is also possible to use 2 rechargeable batteries). Simple and stable placement of the implant, thanks to a unique system of fixation. The Digisonic®SP is the first monobloc implant that integrates a fixation system with 2 titanium self-tapping micro-screws (1); it does not require drilling of the bone or a suture to position the implant. The flexibility of the silicon mounting tabs allows perfect adjustment to the shape of the head. According to a study [7] conducted on the screw fixation system, the unique surgical technique of the Digisonic®SP implant significantly reduces operating time.

Thanks to the deep insertion $(26 \mathrm{~mm})$ of the electrode array into the cochlea, the 20 platinum-iridium electrodes of the Digisonic $₫ S P$ allow stimulation of the complete sound spectrum. Due to the structure of its memory shape and its optimal dimensions, the Digisonic $® S P$ electrode array is recognized by many professionals as the simplest to handle, even in case of anatomic complications. The ease of use of the electrode array of the Digisonic®SP implant favors a natural positioning inside the cochlea.

Cochlear is a medical device company that designs, manufactures and supplies the Nucleus cochlear implant, the Hybrid electro-acoustic implant and the Baha bone conduction implant [8]. Nucleus is a system combining an electrical simulation device that is surgically implanted behind a patient's ear, a processor that captures sounds, and an electrode array that relays the sounds to the brain $[8,9]$. It is a direct descendant of the original cochlear implants, also known as Nucleus, developed by Dr Graeme Clark in Melbourne during the 1970s [10]. Nucleus was the first cochlear implant to be approved by the U.S. Food and Drug Administration [11].

Unique features of Cochlear's electrode portfolio include:

1) 22 active contacts for optimal hearing zone coverage [12], 10 inactive electrodes designed to be inserted closest to the hearing nerve for your best hearing performance $[8,10]$.

2) Best long-term implant reliability record in the industry [13-15]. Electrode placement is critical to hearing performance

Cochlear's electrodes are intended to be placed where hearing nerve stimulation is most effective. In order to benefit from the full range of sound, the area closest to the hearing nerve tends to be stimulated $[9,16]$. This area is known as the hearing zone. Clinical research shows that deeper insertion beyond the hearing zone can be associated with deterioration in performance due to pitch confusion at the tip of the cochlea as well as damage to the delicate cochlear structures $[9,11]$. Insertion depth along with the most active sequential contacts in the industry-22 electrodes [12]-help provide access to the full spectrum of sound and a richer hearing experience. This study is a comparitive study between Oticon Digisonic and Cochlear limited implants outcomes in 200 implant surgeries at late $\operatorname{Dr}$ S N Mehrotra charitable ENT foundation between 2016 to 2019 .

\section{Material and Methods}

Study was carried out at late Dr S N Mehrotra Charitable ENT foundation from 2016 to 2019. 100 children implanted with Digisonic implants and 100 implanted with Cochlear implants were taken into consideration. Study was done by collecting data through fully completed clinical records and information regarding present performance of implantees from our team. The discussion also includes feedback from rehabilitation team about performance of each implantee, the duration at which patients attained speech abilities and reach the respective categories of CAP and SIR score and their GCBI index. The following inclusion criteria were applied to all the selected subjects in the study.

\section{Inclusion criteria}

a) Children with bilateral severe to profound sensori-neural hearing loss.

b) had hearing aid trial for minimum of 3 months

c) psychological assessment was normal.

\section{Evaluation protocol at Mehrotra ENT Hospital}

a) Informed written consent was taken from the parents for the study and follow-ups required during the study.

b) A detailed history and thorough physical and ENT examination was carried out.

c) The subjects then underwent pediatric examination to rule out any neurological condition, which may hamper the child postoperative performance. Prior to implantation a basic workup including hematological, chest X-ray, ECG (electrocardiogram), TORCH (toxoplasmosis, rubella, cytomegalovirus, herpes simplex, and HIV) screen (if require). The general physical condition will be evaluated by anesthetis. A specialist opinion was sought in patients with syndromic etiology of deafness. In children pre implant vaccination will be carried out.

d) Behavioral observational audiometry, impedance, OAE (otoacoustic emissions), auditory brainstem response thresholds and auditory steady-state response was determined to evaluate the degree of hearing loss.

e) Each child was subjected to undergo a high-resolution CT (computed tomography) scan and MRI (magnetic resonance imaging) scan of Temporal bones.

f) Speech perception was also assessed by SIR score before implant 
g) The child was also evaluated by a child psychologist to determine the IQ (intelligent quotient).

h) Counseling of parents was done regarding regular followups and therapy/support to the child at home. They were also made to realize the realistic expectations about the cochlear implant. Also, the parents were made to realize that they are integral part of our rehabilitation team which requires consistent hard work and patience.

Cochlear implantation was done and the impedance checked in Digisonic and neural response telemetry (NRT) was done in nucleus implants and effectiveness assessed in children. Post operatively x-ray was mandatory. The switch on and speech processor tuning done at 1-2 weeks after surgery. Mapping is done at periodic intervals till a stable map is achieved. The rehabilitation program was started out based on baseline skills of child, periodical assessment of outcomes was done in terms of environmental sound speech discrimination and telephonic conversation.

\section{Outcome measures}

a) The subjects were followed up for a maximum period of 1 year at intervals of 3 months, 6 months and 12 months after implantation. Outcome measures were followed as under CAP SCORE, SIR score and GCBI index [17-19].

\section{Results}

Out of 100 children in Digisonic group, 58 were males and 42 females. Out of 100 in cochlear group, 53 were males and 47 females. In Digisonic group, 4 were in age group 6-10 years, 36 belongs to age group of 5-6 years, 10 in age group 4-5 years, 38 to age group 3-4 years and 12 in age group of 1-3 years. In Cochlear group, 5 in age group 6-10 years, 39 in age group of 5-6 years, 11 in age group 4-5 years, 37 in age group 3-4 years and 18 in age group 1-3 years. All children were pre lingual deaf.

\section{Ease of insertion}

The cochlear Ltd implant poses greater difficulty than Digisonic type in insertion. In Cochlear Ltd implants, rings were present which tends to bounce back. A well or a bed has also to be made in cochlear implants. In Digisonic, screws are present to fix it and therefore well or bed was not required to be made. Presence of stopper at the proximal end also helped in stabilizing the implant.

\section{Accessories difference}

In Oticon Digisonic implants, rechargeable battery backup was reported to be poor by parents of children who were using it. Battery once charged fully lasts only for 1-2 hours, sometimes even lesser. Also, the coil connecting processor to the magnet kinks and breaks easily, therefore requiring replacement. In Cochlear LTD. Implants, rechargeable battery backup system is good and works for 12 hours after full recharge. Also coils require less changes.

\section{Comparison of Outcome in Hearing, Speech and Quality of Life}

(Table 1) Cochlear group CAP score was superior compared to Digisonic group. Highest CAP level attained in cochlear group was 10 whereas in Digisonic group 5. 23\% in Cochlear group had CAP level of 5 and above compared to only 1\% in Digisonic group.

(Table 2) 88\% of children in Cochlear group had highest SIR of 5 and 6 compared to $75 \%$ in Digisonic group.

(Table 3) Quality of life index is much better in Cochlear group than Digisonic group. 87\% of cochlear group had GCBI index greater than 80 (maximum benefit) compared to $41 \%$ of Digisonic group. Only 1\% belongs to mild benefit in Cochlear group whereas in Digisonic 29\% in minimal benefit group.88\% of children in Cochlear group had highest SIR of 5 and 6 compared to $75 \%$ in Digisonic group.

Table 1: Comparison of Cap Score of Both Groups.

\begin{tabular}{|c|c|c|}
\hline Cap Score & Digisonic Group & \multicolumn{2}{|c|}{ Cochlear Group } \\
\hline $10-J u l$ & 0 & 7 \\
\hline 6 & 0 & 14 \\
\hline 5 & 1 & 42 \\
\hline 4 & 10 & 21 \\
\hline 3 & 35 & 11 \\
\hline 2 & 53 & 1 \\
\hline
\end{tabular}

Table 2: SIR Score Comparison.

\begin{tabular}{|c|c|c|}
\hline SIR & Digisonic & \multicolumn{2}{|c|}{ Cochlear } \\
\hline 6 & 41 & 47 \\
\hline 5 & 34 & 10 \\
\hline 4 & 17 & 1 \\
\hline 3 & 5 & 10 \\
\hline
\end{tabular}




\begin{tabular}{|l|l|l|}
\hline 2 & 3 & 1 \\
\hline 1 & & \\
\hline
\end{tabular}

Table 3: GCBI.

\begin{tabular}{|c|c|c|}
\hline GCBI & Digisonic & \multicolumn{2}{|c|}{ Cochlear } \\
\hline $91-100$ & 8 & 46 \\
\hline $81-90$ & 33 & 12 \\
\hline $71-80$ & 31 & 1 \\
\hline $61-70$ & 20 & 0 \\
\hline $51-60$ & 7 & 0 \\
\hline$<50$ & 2 & 0 \\
\hline
\end{tabular}

Table 4: Complications.

\begin{tabular}{|c|c|c|}
\hline Complications & Digisonic & Cochlear \\
\hline Device failure & 1 & NONE \\
\hline Hematoma & 3 & 9 \\
\hline Facial paresis & 3 & 4 \\
\hline
\end{tabular}

(Table 4) 1 device failure was seen with Digisonic implants which was later replaced.

Hematoma formation seen more with cochlea, probably owing to formation of well or bed made to fix it.

\section{Services}

Both groups provide equally good service but overall Cochlear LTD. Group is a well-organized team. They regularly conduct training program and seminars for the beneficiary and keeps track of their patients.

\section{Conclusion}

a) Outcome in terms of hearing (CAP), speech (SIR) and quality of life index (GCBI) were better in Cochlear group than Digisonic type.

b) Device accessories complaints were more with Digisonic group owing to poor battery backup.

c) Digisonic implants insertion was easier.

d) Complication such as hematoma were more with Cochlear group owing to drilling for formation of well or bed.

e) Overall Cochlear limited implants were better, thanks to their well-organized team, services, and better outcomes in terms of hearing, speech and quality of life.

\section{Acknowledgement}

None.

\section{Conflict of Interest}

No conflicts of interest.

\section{References}

1. (2012) Oticon A/S: Spaghetti Organization and Beyond". IBS Center for Management Research.
2. (2012) Case Study: Revolution at Oticon A/S: The Spaghetti Organization (Condensed). Harvard Business Review.

3. Peters, Tom (1994) Liberation Management. United States: Ballantine Books. pp:880.

4. Ewing, Jack (2007) Denmark's Masters of E-Mail Marketing. Bloomberg Businessweek.

5. Poulsen, Per Thygesen (1993) Think the Unthinkable: The Revolution in Oticon. Denmark: Schultz Business Books. pp:174.

6. Guevara N, Sterkers 0, Bébéar JP, Meller R, Magnan J, et al. (2010) Multicentre Evaluation of the Digisonic SP Cochlear Implant Fixation System with Titanium Screws in 156 Patients. Annals of Otology, Rhinology \& Laryngology 119: 501-505.

7. Multi Center evaluation of the Digisonic $® S P$ cochlear implant fixation system with titanium screws on 156 patients (Guevara Nicolas MD, Sterkers Olivier MD, PhD, Bébéar Jean-Pierre MD, Meller Renaud MD, Magnan Jacques MD, Mosnier Isabelle MD, Amstutz Isabelle MD, Lerosey Yannick MD, Triglia Jean-Michel MD, Roman Stéphane MD, Gahide Ivan MD).

8. (2015) Jump up to: abcdefghi "Annual report 2015" Cochlear.

9. (2013) The cochlear implant. Powerhouse Museum.

10. (2013) Jump up to: a b "History: Who developed the cochlear implant and why?". Powerhouse Museum.

11. (2013) Cochlear implants technical report. American Speech-LanguageHearing Association.

12. Jump up to: ${ }^{\mathrm{abc}} 2017$ Cochlear Limited Annual Report.

13. (2013) History Cochlear.

14. (2013) Australia's Cochlear plans to return implant to market. Reuters.

15. (2013) Questions mount over Cochlear's top status. 9 News.

16. (2013) Most innovative growth companies. Forbes.

17. Archbold S, Lutman ME, Marshall DH (1995) Categories of Auditory Performance. Ann Otol Rhinol Laryngol 166: 312.

18. Allen C, Nikolopoulos TP, Dyar D (2001) Reliability of a rating scale for measuring speech intelligibility after paediatric cochlear implantation. Otol Neurotol 22: 631-633.

19. Allen C, Nikolopoulos TP, O'Donoghue GM (1998) Speech intelligibility after cochlear implantation. Am J Otol 19: 742-746. 